\title{
新型酞菁-菲分子异质结的合成及其光伏性能研究
}

\author{
俞孝伟 $a, b$ 张山林 ${ }^{b}$ 贺伟伟 ${ }^{b, c}$ 张志刚 ${ }^{b}$ 郭丰启 ${ }^{*, c}$ \\ 詹传郎 ${ }^{b}$ 黄 彦*,a \\ $\left({ }^{a}\right.$ 南京工业大学化学化工学院 材料化学工程国家重点实验室 南京 210009) \\ ( ${ }^{b}$ 中国科学院化学研究所北京分子科学国家实验室 中国科学院光化学重点实验室 北京 100190) \\ ( ${ }^{c}$ 郑州大学化学系 光电功能材料实验室 郑州 450001)
}

\begin{abstract}
摘要 通过酰胺键将酞菁(电子给体单元)和菲二酰亚胺(电子受体单元)偶联, 合成了新型的酞菁-菲分子异质结, 其在 二氯甲烷、氯仿、四氢呋喃等常用溶剂中有较好的溶解度. 紫外光谱分析表明其吸收光谱是唒菁和菲二酰亚胺信号的

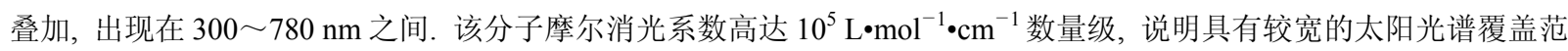
围和很高的吸光系数. 基于这些良好的光谱响应特性, 制备了以该分子与 $[6,6]-$ 苯基- $\mathrm{C}_{61}$-丁酸酸甲酯 $\left(\mathrm{PC}_{61} \mathrm{BM}\right)$ 为光活性 层的有机太阳能电池(OSCs), 该电池器件结构为 ITO/聚(3,4-乙撑二氧噻吩): 聚苯乙烯磺酸(PEDOT:PSS)/酞菁一菲给受体 分子: $\mathrm{PC}_{61} \mathrm{BM} / \mathrm{Ca} / \mathrm{Al}$, 光电转换效率 $(\mathrm{PCE})$ 为 $0.009 \%$, 对应的开路电压 $\left(V_{\mathrm{oc}}\right)$ 为 $0.472 \mathrm{~V}$, 短路电流 $\left(J_{\mathrm{sc}}\right)$ 为 $0.104 \mathrm{~mA} / \mathrm{cm}^{2}$, 填充因子 $(\mathrm{FF})$ 为 0.18 .
\end{abstract}

关键词 酞菁; 菲二酰亚胺; 分子异质结; 光电转换; 有机太阳能电池

\section{Synthesis and Photovoltaic Performances of a Novel Phthalocyanine- perylene Molecular Heterojunction}

\author{
Yu, Xiaowei ${ }^{a, b} \quad$ Zhang, Shanlin $^{b} \quad$ He, Weiwei ${ }^{b, c} \quad$ Zhang, Zhigang $^{b} \quad$ Guo, Fengqi $^{*, c}$ \\ Zhan, Chuanlang ${ }^{b} \quad$ Huang, Yan*,a \\ ( ${ }^{a}$ State Key Laboratory of Materials-Oriented Chemical Engineering, College of Chemistry and Chemical Engineering, \\ Nanjing University of Technology, Nanjing 210009) \\ ( ${ }^{b}$ Beijing National Laboratory for Molecular Sciences (BNLMS), Laboratory of Photochemistry, Institute of Chemistry, \\ Chinese Academy of Sciences, Beijing 100190) \\ ( ${ }^{c}$ Department of Chemistry, Zhengzhou University, Zhengzhou 450001)
}

\begin{abstract}
A novel phthalocyanine (Pc)-perylene diimide (PDI)-Pc donor-acceptor molecular heterojunction was synthesized through amidation of an asymemetric Pc-carboxylic acid and PDI-diamines, and it is well soluble in common solvent such as dichloromethane, chloroform, and tetrahydrofuran. It shows an broad absorption band covering the whole visible region from 300 to $780 \mathrm{~nm}$, and its molar extinction coefficient reaches as high as $10^{5} \mathrm{~L} \cdot \mathrm{mol}^{-1} \cdot \mathrm{cm}^{-1}$. Such an adsorption band originates from both the PDI and PC units. Considering the excellent light-harvesting ability, organic solar cells (OSCs) based on the synthetic compound and [6,6]-phenyl- $\mathrm{C}_{61}$-butyric acid methyl ester $\left(\mathrm{PC}_{61} \mathrm{BM}\right)$ were fabricated with the configurations of ITO/poly(3,4-ethylenedioxythiophene): poly-styrenesulfonate (PEDOT:PSS)/phthalocyanine-perylene: $\mathrm{PC}_{61} \mathrm{BM} / \mathrm{Ca} / \mathrm{Al}$. One of the OSC devices showed a power conversion efficiency (PCE) of $0.009 \%$ with an open-circuit voltage $\left(V_{\text {oc }}\right)$ of $0.472 \mathrm{~V}$, a short circuit current $\left(J_{\mathrm{sc}}\right)$ of $0.104 \mathrm{~mA} / \mathrm{cm}^{2}$ and a fill factor $(\mathrm{FF})$ of $18.2 \%$.
\end{abstract}

Keywords phthalocyanine; perylene dimide; molecular heterojuntion; photon-to-electron conversion; organic solar cell

菲二酰亚胺、卟啉、酞菁等是含有大 $\pi$ 共轭体系的 染料化合物, 由于它们具有优异的光学和电子学性质,
在化学和材料科学领域倍受关注. 菲二酰亚胺(PDI)在 光、热等条件下均十分稳定，其单体具有很高的苂光量

\footnotetext{
*E-mail: huangy@njut.edu.cn

Received March 31, 2012; revised May 27, 2012; published online June 4, 2012.

Project supported by the National Natural Science Foundation of China (No. 20973182).

国家自然科学基金(No. 20973182)资助项目.
} 
子产率 $(0.9)^{[1 \sim 6]}$. 尤其值得注意的是, 它们还是一类典 型的 $n$-型有机半导体, 可以用作有机太阳能电池(OSCs) 中的电子受体材料 ${ }^{[7 \sim 11]}$, 有机场效应晶体管(OFETs)和 有机薄膜晶体管(OTFTs)中的电子传输材料 ${ }^{[12 \sim 16]}$. 同样, $\mathrm{p}$-型有机半导体酞菁以其独特的化学稳定性和优异的 光学特征 ${ }^{[17,18]}$, 自发现以来一直受到人们的关注. 从最

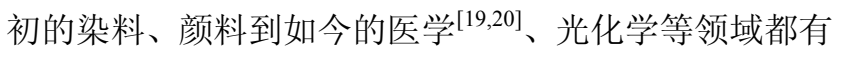
广泛的应用..$^{21 \sim 25]}$

菲二酰亚胺的吸收范围在 $350 \sim 620 \mathrm{~nm}$, 酞菁的吸 收范围在 $280 \sim 450$ 和 $580 \sim 780 \mathrm{~nm}$, 可见, 菲二酰亚胺 和酞菁的吸收峰具有很强的互补特性, 两者的组合可以 覆盖太阳光谱的可见光谱区域. 本研究在含羒基的不对 称酞菁基础上 ${ }^{[26]}$, 以 1,6,7,12-四氯-3,4:9,10-菲二酸酕为 原料合成两端含氨基的 $N, N^{\prime}$-二(3-氨基丙基)-1,6,7,12-四 (4-叔丁基苯氧基)-3,4:9,10-芢二酰亚胺, 通过酰胺键合 成了酞菁-菲-酞等分子异质结(Schemes $1 \sim 2$, 该分子 异质结具有较宽的吸收光谱, 有较好的溶解性, 可通过 溶液加工方法制备体异质结型有机太阳能电池.

\section{1 实验部分}

\section{1 实验仪器及药品}

Bruker 300, 400 或 $600 \mathrm{~Hz}$ 核磁共振仪(内标 TMS, 溶剂为氛代氯仿 $\mathrm{CDCl}_{3}$ 或気代二甲亚砜 $d_{6}$-DMSO); Bruker Biflex III 飞行时间质谱仪 (MALTI-TOF) 或 (ESI-MS); 紫外-可见吸收光谱仪 (Lamda25, PerkinElmer); 荧光光谱仪 (Lamda25, Perkin-Elmer); 德国 Zahner IM6e 电化学工作站; Millipore-Q 纯水机; AP $250 \mathrm{D}$ 分析天平; 原子力显微镜(PicoPlus, MI); 柱层析硅 胶产自青岛海洋化工厂 (200 300 目); 所用化学试剂均 为分析纯.

$1.2 N, N^{\prime}$-二(3-氨基丙基)-1,6,7,12-四(4-叔丁基苯氧 基)-3,4:9,10-菲二酰亚胺的合成

1.2.1 $N, N^{\prime}$-二(2-乙基已基)-1,6,7,12-四氯-3,4:9,10-芢 二酰亚胺(2)的合成

将 1,6,7,12-四氯-3,4:9,10-菲二酸酮(1) $10.0 \mathrm{~g}, 2$-乙 基己胺 $9.40,150 \mathrm{~mL}$ 的异丙醇加入 $250 \mathrm{~mL}$ 的三口烧瓶 中, 在 $\mathrm{N}_{2}$ 保护下 $87{ }^{\circ} \mathrm{C}$ 回流, $10 \mathrm{~h}$ 后停止反应. 反应液冷 却至室温, 倒入 $200 \mathrm{~mL}$ 水中, 搅拌后过滤, 用 $5 \%$ 的 $\mathrm{NaOH}$ 水溶液洗涤, 再用水洗至中性, 得到红色固 体 ${ }^{[27,28]}$, 干燥, 得产品 $9.1 \mathrm{~g}$, 产率 $90.1 \% .{ }^{1} \mathrm{H}$ NMR $\left(\mathrm{CDCl}_{3}, 400 \mathrm{MHz}\right) \delta: 8.68(\mathrm{~s}, 4 \mathrm{H}), 4.10 \sim 4.21(\mathrm{~m}, 4 \mathrm{H})$, $1.94(\mathrm{t}, J=8.0 \mathrm{~Hz}, 2 \mathrm{H}), 1.40(\mathrm{t}, J=8.0 \mathrm{~Hz}, 8 \mathrm{H}), 1.35$ (d, $J=8.0 \mathrm{~Hz} 8 \mathrm{H}), 0.90 \sim 0.97(\mathrm{~m}, 12 \mathrm{H})$; TOF-MS $m / z(\%)$ : $752.1\left(\mathrm{M}^{+}, 100\right)$.
1.2.2 $N, N^{\prime}$-二(2-乙基已基)-1,6,7,12-四(4-叔丁基苯氧 基)-3,4:9,10-萠二酰亚胺(3)的合成

将 $5.00 \mathrm{~g}(2), 9.75 \mathrm{~g}$ 对叔丁基苯酚, $8.97 \mathrm{~g} \mathrm{~K}_{2} \mathrm{CO}_{3}$ 加 入到盛有 $80 \mathrm{~mL} N$-甲基吡咯烷酩(NMP)的三口烧瓶中, 在 $\mathrm{N}_{2}$ 保护下加热至 $130{ }^{\circ} \mathrm{C}$, 反应 $10 \mathrm{~h}$ 后冷却至室温. 倒入 $500 \mathrm{~mL}$ 体积比为 $10 \%$ 盐酸水溶液中摚拌过滤. 用 水洗至中性, 干燥, 得红色固体 $7.75 \mathrm{~g}^{[29,30]}$, 产率 $90.8 \%$. ${ }^{1} \mathrm{H} \mathrm{NMR}\left(\mathrm{CDCl}_{3}, 400 \mathrm{MHz}\right) \delta: 8.23$ (s, 4H), 7.25 (d, $J=$ $8.0 \mathrm{~Hz}, 8 \mathrm{H}), 6.83$ (d, $J=8.0 \mathrm{~Hz}, 8 \mathrm{H}), 4.15(\mathrm{t}, J=8.0 \mathrm{~Hz}$, $4 \mathrm{H}), 1.85$ (s, 2H), 1.56 (s, 8H), 1.29 (s, 36H), 1.28 (s, 8H), $0.83 \sim 0.90$ (m, 12H); TOF-MS $m / z(\%): 1207.5\left(\mathrm{M}^{+}, 100\right)$. 1.2.3 1,6,7,12-四(4-叔丁基苯氧基)-3,4:9,10-芢二酸 酐(4)的合成

将 $5.00 \mathrm{~g} \mathrm{(3),} 3.0 \mathrm{~g} \mathrm{KOH}$ 和 $15 \mathrm{~mL} \mathrm{H}_{2} \mathrm{O}$ 加入到 350 $\mathrm{mL}$ 异丙醇中, $\mathrm{N}_{2}$ 保护回流过夜, 溶液由原来的红色变 为绿色, 反应后将溶液冷却至室温, 将反应液倒入 500 $\mathrm{mL}$ 体积比 $10 \%$ 的盐酸水溶液中搅拌, 有红色固体析出, 过滤, 水洗至中性, 干燥, 得到暗红色固体 $3.8 \mathrm{~g}^{[31]}$. 将 固体产物溶于少量氯仿, 用柱层析提纯, 纯氯仿作为淋 洗液. 得产物 $2.3 \mathrm{~g}$, 产率 52.9\%. ${ }^{1} \mathrm{H}$ NMR $\left(\mathrm{CDCl}_{3}, 400\right.$ MHz) $\delta$ : 8.23 (s, 4H), 7.25 (d, $J=8.0 \mathrm{~Hz}, 8 \mathrm{H}), 6.83$ (d, $J=$ $8.0 \mathrm{~Hz}, 8 \mathrm{H}), 1.30$ (s, 36H); TOF-MS $m / z(\%): 985.1\left(\mathrm{M}^{+}\right.$, 100).

1.2.4 $N, N^{\prime}$-二(3-Boc-氨基丙基)-1,6,7,12-四(4-叔丁基 苯氧基)-3,4:9,10-菲二酰亚胺(5)的合成

将 $2.0 \mathrm{~g}(4), N$-Boc-氨基丙胺 $5 \mathrm{~mL}, \mathrm{DMF} 50 \mathrm{~mL}$, 咪 唑 $10 \mathrm{~mL}$, 加入于 $250 \mathrm{~mL}$ 的三口烧瓶中, 在 $\mathrm{N}_{2}$ 保护下 $87{ }^{\circ} \mathrm{C}$ 回流, $10 \mathrm{~h}$ 后停止反应. 把反应液冷却至室温, 倒 入 $200 \mathrm{~mL}$ 水中, 摚拌后过滤, 用 $5 \%$ 的 $\mathrm{NaOH}$ 水溶液洗 涤, 再用去离子水洗至中性, 得到红色固体 ${ }^{[32,33]}$, 干燥. 得产物 $1.3 \mathrm{~g}$, 产率 62.1\%. ${ }^{1} \mathrm{H} \mathrm{NMR}\left(\mathrm{CDCl}_{3}, 400 \mathrm{MHz}\right) \delta$ : $8.22(\mathrm{~s}, 4 \mathrm{H}), 7.25$ (d, $J=8.0 \mathrm{~Hz}, 8 \mathrm{H}), 6.83(\mathrm{~d}, J=8.0 \mathrm{~Hz}$, $8 \mathrm{H}), 5.11(\mathrm{~s}, 2 \mathrm{H}), 4.19(\mathrm{t}, J=8.0 \mathrm{~Hz}, 4 \mathrm{H}), 3.11(\mathrm{t}, J=8.0$ $\mathrm{Hz}, 4 \mathrm{H}), 1.86 \sim 1.88(\mathrm{~m}, 4 \mathrm{H}), 1.41(\mathrm{~s}, 18 \mathrm{H}), 1.30(\mathrm{~s}, 36 \mathrm{H})$; TOF-MS $m / z(\%): 1264.6\left(\mathrm{M}^{+}, 100\right)$.

1.2.5 $N, N^{\prime}$-二(3-氨基丙基)-1,6,7,12-四(4-叔丁基苯 氧基)-3,4:9,10-菲二酰亚胺(6) 的合成

将 $1.0 \mathrm{~g}(\mathbf{5})$ 溶于 $20 \mathrm{~mL}$ 二氯甲烷, 加入 $1 \mathrm{~mL}$ 三氟乙 酸, 常温傥拌 $5 \mathrm{~h}$, 加入 $30 \mathrm{~mL} \mathrm{Na} \mathrm{CO}_{3}$ 的水溶液, 用二 氯甲烷/水反复萃取, 取有机层, 旋干, 得到深红色固体, 干燥, 得产物 $0.9 \mathrm{~g}$, 产率 88.7\%. ${ }^{1} \mathrm{H}$ NMR (DMSO- $d_{6}$, $400 \mathrm{MHz}) \delta: 7.92$ (s, 4H), 7.34 (d, $J=8.0 \mathrm{~Hz}, 8 \mathrm{H}), 6.90$ (d, $J=8.0 \mathrm{~Hz}, 8 \mathrm{H}), 4.02(\mathrm{t}, J=8.0 \mathrm{~Hz}, 4 \mathrm{H}), 2.73$ (t, $J=8.0$ $\mathrm{Hz}, 4 \mathrm{H}), 1.81 \sim 1.82(\mathrm{~m}, 4 \mathrm{H}), 1.26$ (s, 36H); TOF-MS $\mathrm{m} / \mathrm{z}$ 

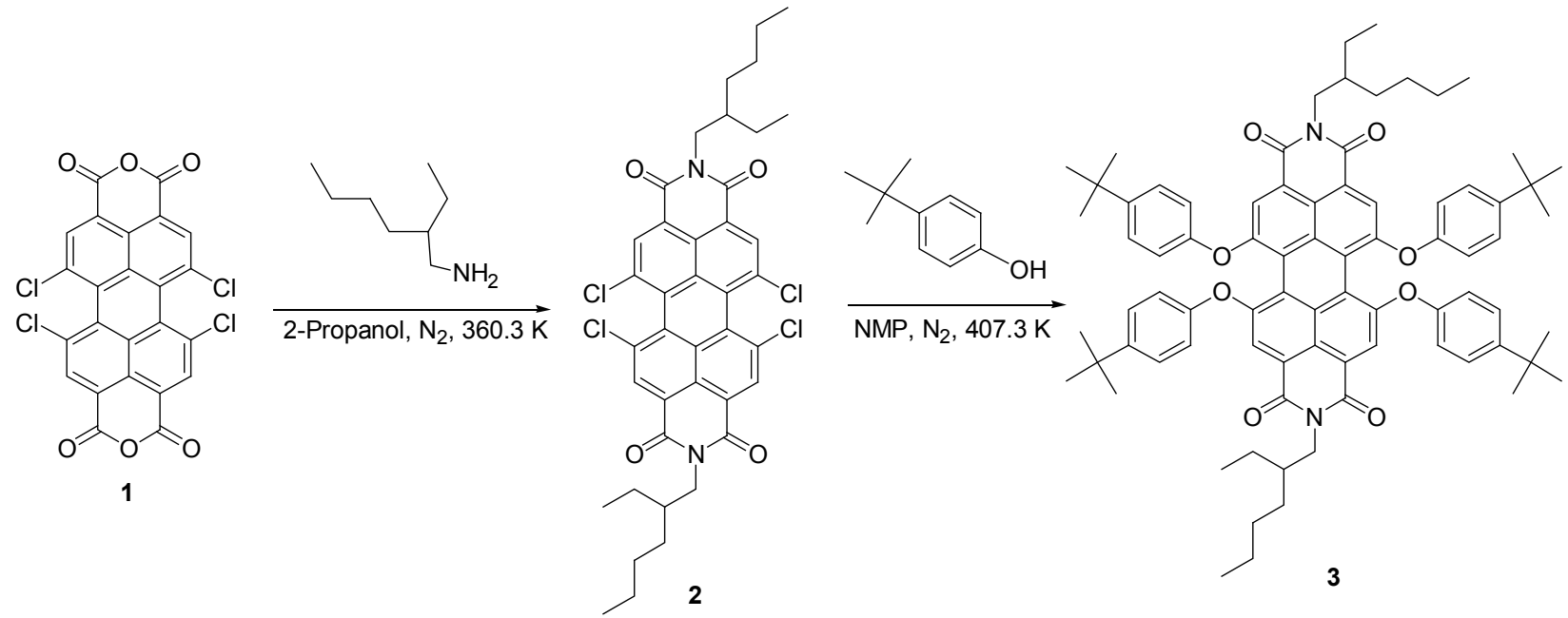

3

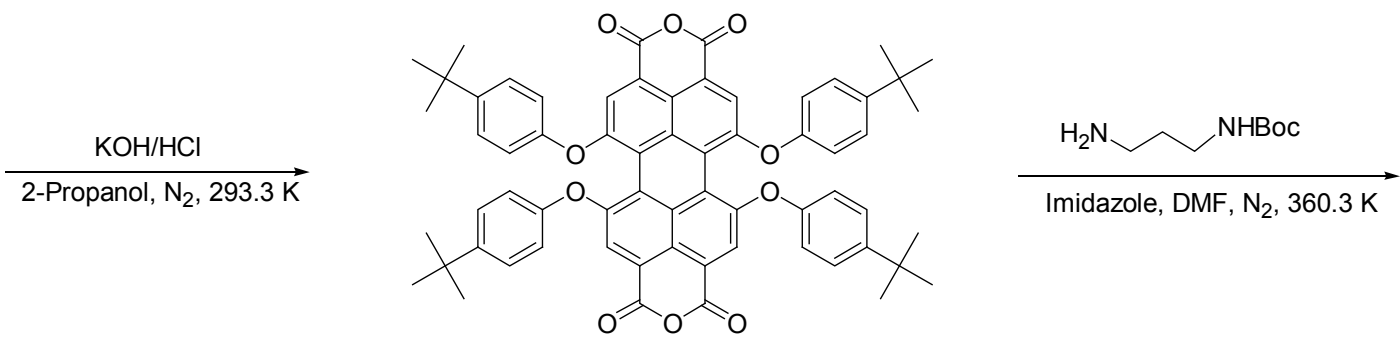

4<smiles>CC(C)(C)OC(=O)NCCCN1C(=O)c2cc(Oc3ccc(C(C)(C)C)cc3)c3c4c(Oc5ccc(C(C)(C)C)cc5)cc5c6c(cc(Oc7ccc(C(C)(C)C)cc7)c(c7c(Oc8ccc(C(C)(C)C)cc8)cc(c2c37)C1=O)c64)C(=O)N(CCCNC(=O)OCc1ccccc1)C5=O</smiles>

(\%): $1097.4\left(\mathrm{M}^{+}, 100\right)$.

\section{3 酞菁-菲-酞菁分子异质结的合成}

2(3),9(10),16(17)-三-(对叔丁基苯氧基)-23(24)-(丁 二酸单乙酯酰胺基)-酞菁锌(7)和 2(3),9(10),16(17)-三(对叔丁基苯氧基)-23(24)-(丁二酸单酸酰胺基)-酞菁锌 (8)的合成见文献[26].

1.3.1 酞菁一蒈一酞箐分子异质结:Pc-PDI-Pc (9)的合 成

取化合物 $80.25 \mathrm{~g}$, 化合物 $60.1 \mathrm{~g}, 1$-羟基苯并三唑

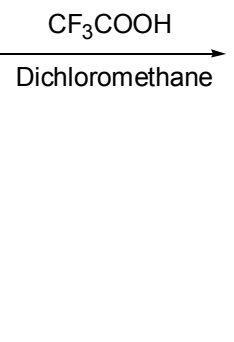

Scheme 1

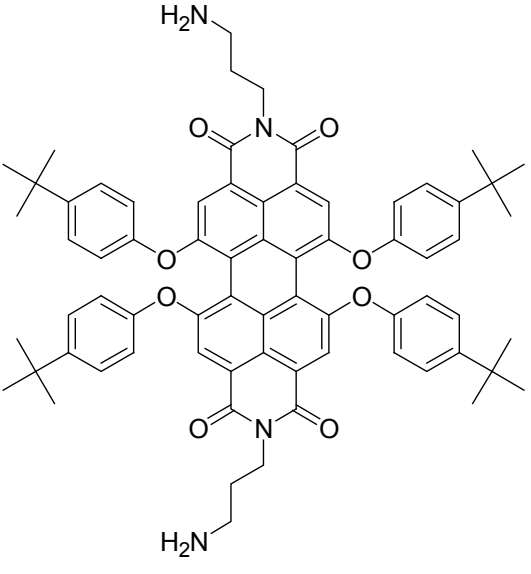

(HOBT) $0.1 \mathrm{~g}, N, N$-二异丙基乙胺(DIEA) $0.1 \mathrm{~mL}$, 先将 化合物 8, HOBT, DIEA 溶于 THF, 冰浴下搅拌 $0.5 \mathrm{~h}$, 再 加入化合物 6 , 摚拌至室温后反应 $5 \mathrm{~h}$. 反应结束后, 以 二氯甲烷/水萃取，取有机相，拌硅胶旋干后，柱层析分 离: (a)先用二氯甲烷淋洗至淋洗液无色; (b)用二氯甲烷/ 乙酸乙酯 $(V: V=10: 1)$ 淋洗至淋洗液无色; (c)用二氯 甲烷/乙酸乙酯 $(V: V=1: 1)$ 淋洗得到双边产物 $\mathbf{9}^{[34]} ;(\mathrm{d})$ 用二氯甲烷/甲醇 $(V: V=5: 1)$ 淋洗得到单边产物 0.18 $\mathrm{g}$, 产率 $49.7 \%$. 

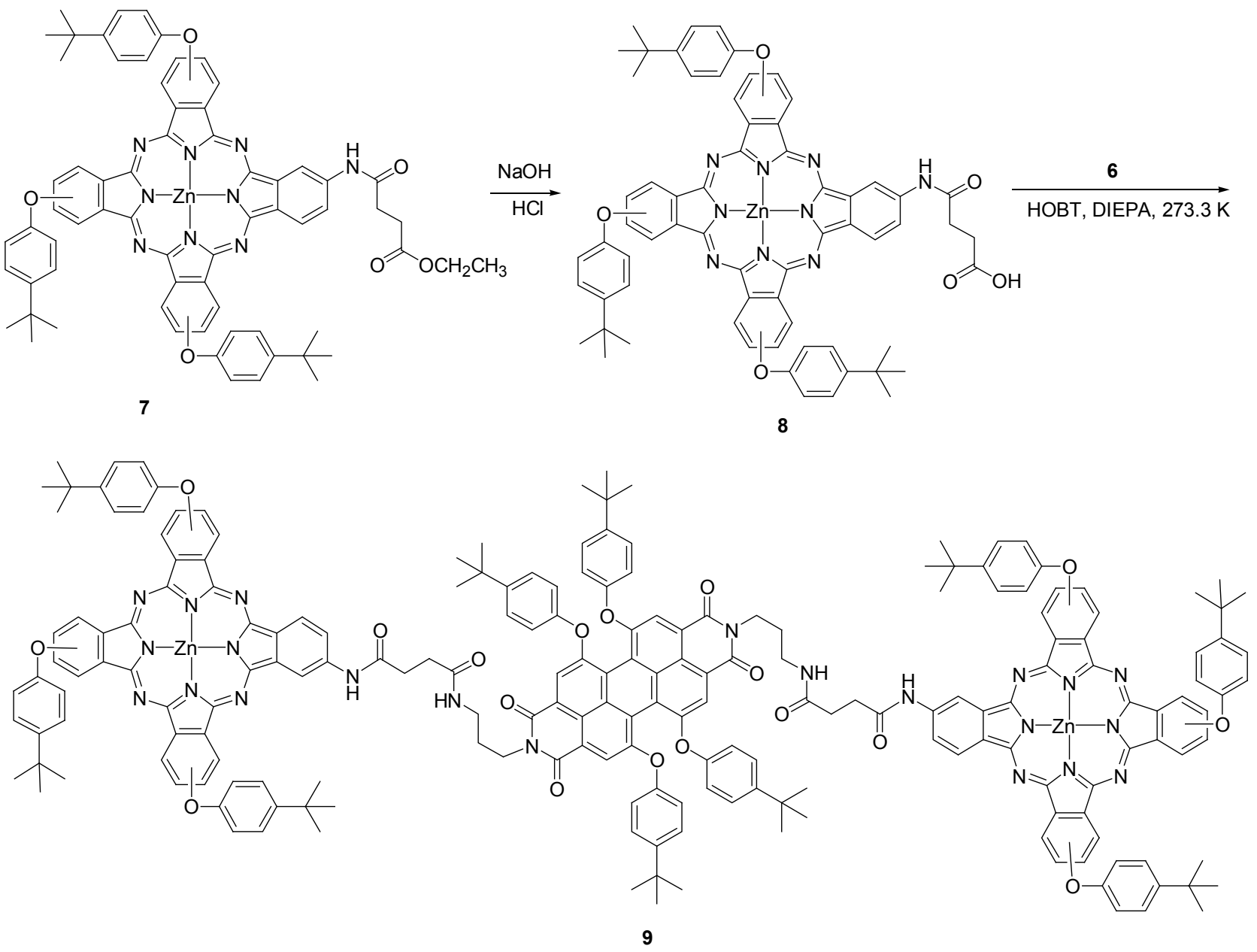

Scheme 2

1.3.2 酞菁-菲一酞箐分子异质结:Pc-PDI-Pc (9)的分 析数据

${ }^{1} \mathrm{H}$ NMR $\left(d_{6}\right.$-DMSO, $\left.400 \mathrm{MHz}\right) \delta: 8.25 \sim 9.30(\mathrm{~m}$, $20 \mathrm{H}), 7.72 \sim 7.81(\mathrm{~m}, 20 \mathrm{H}), 7.57 \sim 7.70(\mathrm{~m}, 12 \mathrm{H}), 7.30 \sim$ $7.40(\mathrm{~m}, 12 \mathrm{H}), 6.82(\mathrm{~d}, J=8.0 \mathrm{~Hz}, 8 \mathrm{H}), 6.79 \sim 6.81(\mathrm{~m}$, $4 \mathrm{H}), 4.07$ (s, 4H), $2.91(\mathrm{~s}, 4 \mathrm{H}), 2.65(\mathrm{~s}, 4 \mathrm{H}), 1.85(\mathrm{~s}, 4 \mathrm{H})$, $1.38(\mathrm{~d}, J=12 \mathrm{~Hz}, 54 \mathrm{H}), 1.31$ (s, 36H); TOF-MS $m / z(\%)$ : $3333.4\left(\mathrm{M}^{+}, 100\right)$; HRMS calcd for $\mathrm{C}_{202} \mathrm{H}_{182} \mathrm{~N}_{22} \mathrm{O}_{18} \mathrm{Zn}_{2}$ 1666.63787, found 1666.63655 .

\section{4 光谱测试}

将化合物 5, 6 和 9 溶于 THF，配成 $5 \times 10^{-4} \mathrm{~mol} / \mathrm{L}$ 的 THF 溶液, 采用厚度为 $0.1 \mathrm{~cm}$ 的比色具对所配样品 进行紫外测试. 根据兰伯特比尔定律: $\lg A=\varepsilon c l$, 计算出 溶液态的摩尔消光系数, 其中 $A$ 为吸光度, $\varepsilon$ 为摩尔消光 系数, 单位为 $\mathrm{L} \cdot \mathrm{mol}^{-1} \cdot \mathrm{cm}^{-1}, c$ 为溶液浓度, 单位为 $\mathrm{mol} / \mathrm{L}, l$ 为光程, 即比色㿼厚度, 单位为 $\mathrm{cm}$. 采用厚度 为 $1 \mathrm{~cm}$ 的比色典对所配样品进行荧光测试.

\section{5 电化学测试}

电化学循环伏安曲线的测定采用德国 Zahner IM6e 电化学工作站和三电极体系, 把玻碳电极作为工作电 极, 铂丝作对电极, $\mathrm{Ag} / \mathrm{AgCl}$ 作参比电极, 六氟磷酸四丁 基铵 $\left(\mathrm{Bu}_{4} \mathrm{NPF}_{6}\right)$ 作为电解, 将分子 9 溶解在 $0.1 \mathrm{~mol} / \mathrm{L}$ 的 $\mathrm{Bu}_{4} \mathrm{NPF}_{6}$ 的四氢呋喃 $(\mathrm{THF})$ 溶液中, 9 的浓度为 $10^{-4}$ $\mathrm{mol} / \mathrm{L}$. 将电极插入溶液中进行测定. 根据氧化/还原峰 的起始电位估算出 Pc-PDI-Pc 的的 HOMO(最高占据分 子轨道)和 $\operatorname{LUMO}$ (最低末占据分子轨道)能级.

\section{6 太阳能电池制备}

采用 ITO 玻璃作为衬底, 刻蚀后, 依次用去离子. 水、丙酮、乙醇、去离子水超声清洗，每次超声时间为 $20 \mathrm{~min}$; 氮气吹干, 将 ITO 玻璃进行 $20 \mathrm{~min}$ 的紫外-臭氧 $\left(\mathrm{UV} / \mathrm{O}_{3}\right)$ 处理; 以 $2000 \mathrm{r} / \mathrm{min}$ 的速度旋涂 PEDOT:PSS, 旋涂时间为 $35 \mathrm{~s}$, 之后在 $150{ }^{\circ} \mathrm{C}$ 下烘干 $15 \mathrm{~min}$. 有机太 阳能电池的活性层选取 PC-PDI-PC (9):PCBM 的 THF 溶 液 $(10 \mathrm{mg} / \mathrm{mL})$, 分别以 $800,1500,2000 \mathrm{r} / \mathrm{min}$ 的速度旋 涂, 旋涂时间为 $30 \mathrm{~s}$. 在高真空环境 $\left(5.5 \times 10^{-5} \mathrm{~Pa}\right)$ 下进 
行蒸镀成膜, 所制备的电池的有效面积为 $2 \times 3 \mathrm{~mm}^{2}$. 用 光强为 $100 \mathrm{~mW} / \mathrm{cm}^{2}$ 太阳光模拟光源, 在氮气环境下进 行 $J-V$ 性能测试.

\section{7 有机活性层薄膜的形貌表征}

采用 ITO 玻璃作为基底, 经过与第 1.4 节相同的清 洗及 PEDOT:PSS 修饰处理, 以 $800 \mathrm{r} / \mathrm{min}$ 的速度分别旋 涂 PDI-PC-PDI (9): $\mathrm{PC}_{61} \mathrm{BM}$ 的 THF 溶液和聚-3-己基噻吩 $(\mathrm{P} 3 \mathrm{HT}): \mathrm{PCB}_{61} \mathrm{M}$ 的 1-氯苯溶液, 旋涂时间为 $30 \mathrm{~s}$. 选用 美国 MI 公司的 PicoPlus 型号原子力显微镜(AFM)观察 两者的形貌, 进行对比.

\section{2 结果与讨论}

\section{1 合成部分}

本文从溶解性较好的 $N, N$-二(2-乙基已胺)-1,6,7,12四氯-3,4:9,10-芢二酸䣶出发,在腰位修饰 4-叔丁基苯酚 后, 得到 4-叔丁基苯氧取代基的衍生物, 然后水解, 得 到化合物 4 再合成得到化合物 $\mathbf{5}$. 因为 $1,6,7,12$-四氯3,4:9,10-芢二酸䣶用 2-乙基已胺酰胺化后, 溶解性显著 提高, 分离提纯比较容易, 可降低后续合成产物分离纯 化的难度和提高产率. 在合成化合物 9 时, 我们采用了 常用的活化酸法, 以 HOBT 为缩合剂, 在投料过程中选 择酞菁化合物 8 的投料比过量 $0.2 \sim 0.4$ equiv., 这样尽 量避免菲二酰亚胺的单边取代的化合物生成, 降低提纯 时的难度, 同时也提高菲二酰亚胺双边取代产物即化合 物 9 的产率.

\section{2 光物理性质}

如图 1 所示, 紫外吸收光谱表明, 酞菁分子 7 的吸 收范围在: $300 \sim 45$ 和 $600 \sim 750 \mathrm{~nm}$ 之间, 摩尔消光系数

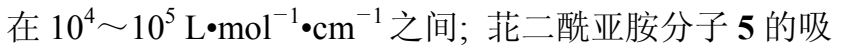
收范围在: $360 \sim 600 \mathrm{~nm}$ 之间, 摩尔消光系数达到 $10^{4}$ $\mathrm{L} \cdot \mathrm{mol}^{-1} \cdot \mathrm{cm}^{-1}$, 所设计酞菁-菲二酰亚胺分子 9 光谱覆盖 范围在 300 750 nm 之间, 且摩尔消光系数也在 $10^{4} \sim$ $10^{6} \mathrm{~L} \cdot \mathrm{mol}^{-1} \cdot \mathrm{cm}^{-1}$ 之间. 可以看出分子 9 的吸收光谱是 分子 5 和分子 7 的吸收光谱的叠加, 同时也证明了分子 9 中有酞菁和菲二酰亚胺单元的存在. 同样, 如图 2 中所 示, 唒菁(5)苂光激发光谱在 700 $800 \mathrm{~nm}$ 之间; 菲二酰 亚胺(7)荧光激发光谱在 $550 \sim 700 \mathrm{~nm}$ 之间. 所设计酞菁 -菲二酰亚胺分子 9 在 550 700 和 $700 \sim 800 \mathrm{~nm}$ 之间都 有的荧光激发光谱, 从而进一步的证明了分子 9 中有酞 菁和芢二酰亚胺单元的存在.

\section{3 电化学性能}

图 3 是 Pc-PDI-Pc (9)的循环伏安曲线图, 电解液为 $0.1 \mathrm{~mol} / \mathrm{L} \mathrm{Bu} 4 \mathrm{NPF} 6$ 的 THF 溶液. 从图 3 可以看出, 化 合物 9 氧化起始电位为 $0.67 \mathrm{~V}$, 根据公式 $E_{\mathrm{HOMO} / \mathrm{LUMO}}=$

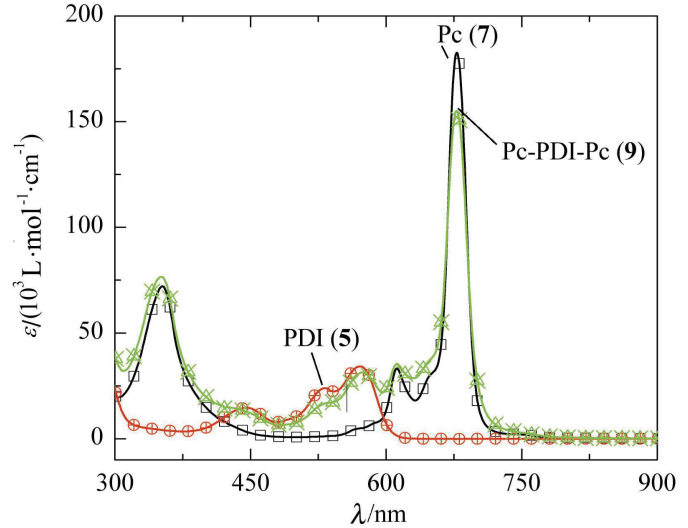

图 1 化合物 5, 7 和 9 的四氢呋喃溶液的紫外吸收光谱 Figure 1 UV-vis absorption spectra of the compounds 5, 7 and 9 in THF

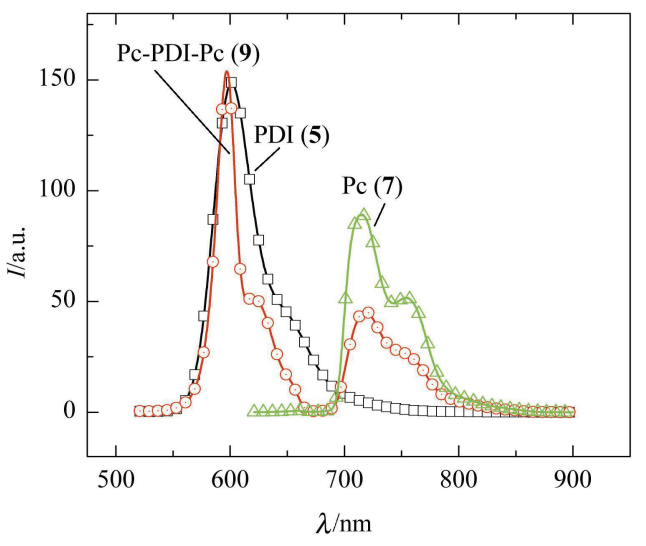

图 2 化合物 5,7 和 9 的四氢呋喃溶液的苂光光谱 Figure 2 Fluorescence spectra of compounds 5, 7 and 9 in THF $-\left(E_{\text {onset(ox } / \mathrm{re})}+4.40\right) \mathrm{eV}^{[35]}$, 得出 Pc-PDI-Pc (9)的 HOMO 为 $-5.07 \mathrm{eV}$, 较低的 $\mathrm{HOMO}$ 能级能确保材料有较高的 空气稳定性. 化合物 9 还原起始电位为 -1.04 , 则 LUMO 为 $-3.36 \mathrm{eV}$. 如图 3 中所给出的 Pc-PDI-Pc (9) 的 HOMO 和 LUMO 与 $\mathrm{PC}_{61} \mathrm{BM}$ 的 HOMO, LUMO 的能 级结构匹配图. 根据相关文献[36]报道, 给体的 LUMO 能级比受体的 LUMO 能级高出 $0.3 \mathrm{eV}$ 以上才能保证给 受体之间有效激子分离. 化合物 9 的 LUMO 比 PCBM 的高出 0.55 , 制成异质结活性层可以保证有效的激子分 离.

\section{4 光伏性能}

在有机太阳能电池 ITO/PEDOT:PSS $(30 \mathrm{~nm}) / \mathrm{Pc}$ PDI-Pc (9): $\mathrm{PC}_{61} \mathrm{BM} / \mathrm{Ca}(12 \mathrm{~nm}) / \mathrm{Al}(160 \mathrm{~nm})$ 的制备中, 我们选取 Pc-PDI-Pc 为电子给体材料, $\mathrm{PC}_{61} \mathrm{BM}$ 为受体材 料, 通过溶液旋涂的方法制备光活性层. 同时, 在电池 的制备过程中, 我们分别以 Pc-PDI-Pc (9)： $\mathrm{PC}_{61} \mathrm{BM}$ 为 $1.0: 0.5,1.0: 1.0,1.0: 2.0$ 三种比例 $(10 \mathrm{mg} / \mathrm{mL})$ 的 $\mathrm{THF}$ 


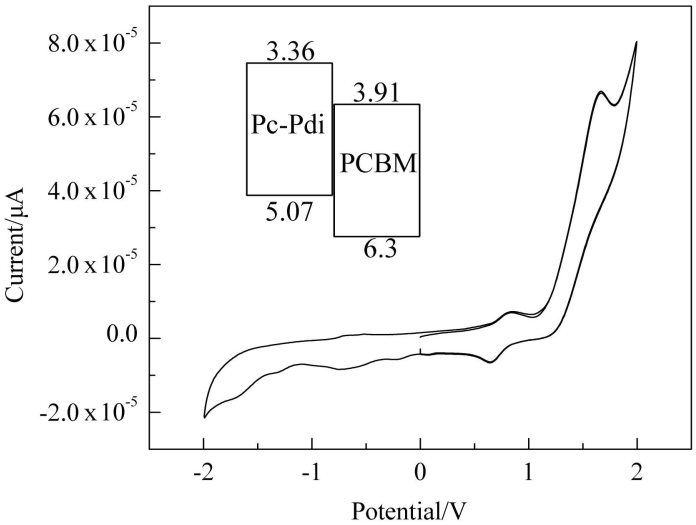

图 3 Pc-PDI (9) 在 $0.1 \mathrm{~mol} / \mathrm{L} \mathrm{Bu}_{4} \mathrm{NPF}_{6}$ 的 $\mathrm{THF}$ 溶液的循环伏 安图及化合物 9 和 $\mathrm{PCBM}$ 的能级图

Figure 3 Cyclic voltammograms of Pc-Pdi (9) in the $0.1 \mathrm{~mol} / \mathrm{L}$ $\mathrm{Bu}_{4} \mathrm{NPF}_{6} \mathrm{THF}$ solution and Energy-level diagram between compounds 9 and PCBM

溶液, 和 $800,1500,2000 \mathrm{r} / \mathrm{min}$ 三种速度来太阳能电池 的活性层. 如表 1 所示, 在所制成的电池中, 开路电压 都能够达到 $0.4 \mathrm{~V}$, 而电池的短路电流在 $0.05 \sim 0.10$ $\mathrm{mA} / \mathrm{cm}^{2}$, 填充因子也在 0.2 左右, 在所制备的电池中 Pc-PDI-Pc (9): $\mathrm{PC}_{61} \mathrm{BM}$ 的重量比为 $1.0: 0.5$, 光活性层 旋涂速度为 $800 \mathrm{r} / \mathrm{min}$ 时表现出 $0.009 \%$ 的最高的转换效 率，图 4 所示为该条件下的 $J$ (Photocurrent density)- $V$ (voltage) 曲线, 对应的开路电压为 $0.472 \mathrm{~V}$, 短路电流为 $0.104 \mathrm{~mA} / \mathrm{cm}^{2}$, 填充因子为 0.18 . 开路电压 $\left(V_{\mathrm{oc}}\right)$ 与给体 的 HOMO 和受体的 LUMO 相关联, 由于化合物 9 的 $\mathrm{HOMO}$ 能级较高, 因此 $V_{\mathrm{oc}}$ 较低. 在所制备的有机太阳 能电池中, 均表现出较低的短路电流和较差的填充因 子. 主要在制备电池过程中发现 Pc-PDI-Pc (9)/ $/ \mathrm{PC}_{61} \mathrm{BM}$ 的活性材料的成膜性不够好, 在旋涂的过程中, 经常会 出现膜的不均匀或小颗粒形成. 较差的成膜性严重影响 了载流子的传输, 这可能是导致短路电流和填充因子偏 低的重要原因, 这将会在成膜性研究中进一步的讨论.

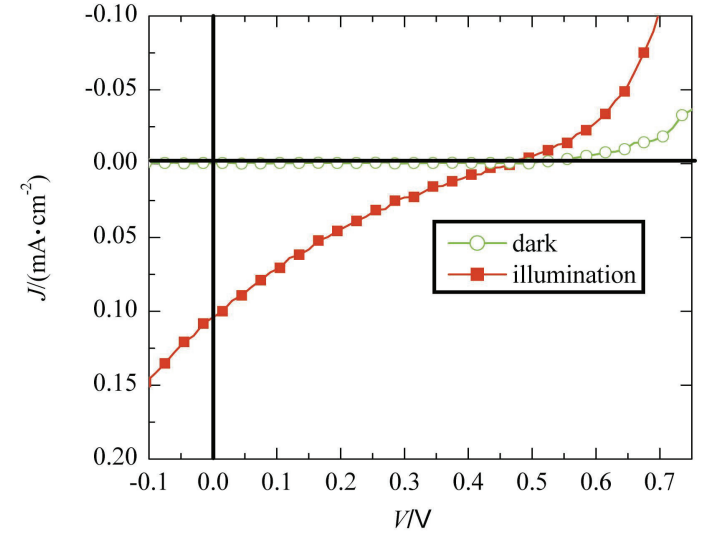

图 4 Pc-PDI-Pc (9): $\mathrm{PC}_{61} \mathrm{BM}$ 组成的 $\mathrm{OSC}$ 的 $J-V$ 曲线 Figure 4 Photocurrent density $(J)$-voltage $(V)$ characteristic of OSC with Pc-PDI-Pc (9): $\mathrm{PC}_{61} \mathrm{BM}$

\section{5 成膜性研究}

如图 5 所示, 相同条件下的 Pc-PDI-Pc/ $/ \mathrm{PC}_{61} \mathrm{BM}$ 和 $\mathrm{P} 3 \mathrm{HT} / \mathrm{PC}_{61} \mathrm{BM}$ 的 $\mathrm{AFM}$ 图: 图 $5 \mathrm{a}$ 和图 $5 \mathrm{~b}$ 为 $\mathrm{Pc}-\mathrm{PDI} /$ $\mathrm{PC}_{61} \mathrm{BM}$ 的平面图和立体图, 图 $5 \mathrm{c}$ 和图 $5 \mathrm{~d}$ 为 $\mathrm{P} 3 \mathrm{HT} /$ $\mathrm{PC}_{61} \mathrm{BM}$ 的平面图和立体图. 对 $\mathrm{Pc}-\mathrm{PDI}-\mathrm{Pc} / \mathrm{PC}_{61} \mathrm{BM}$ 的成 膜性进行研究对比, 从图 5a 图中可以看出 Pc-PDI-Pc/ $\mathrm{PC}_{61} \mathrm{BM}$ 膜的粗粘度达到 $380 \mathrm{~nm}$, 且呈现出比较大的颗 粒，而在 P3HT/ $\mathrm{PC}_{61} \mathrm{BM}$ 膜的起伏度只有 $10 \mathrm{~nm}$ ，且膜的 表面较为平整(图 5c). 对比图 $5 \mathrm{~b}$ 和图 $5 \mathrm{~d}$ 的三维图也可 以得到相同的结论, $\mathrm{P} 3 \mathrm{HT} / \mathrm{PC}_{61} \mathrm{BM}$ 作为经典的有机太阳 能电池活性层组合 ${ }^{[37]}, \mathrm{Pc}-\mathrm{PDI} / \mathrm{PC}_{61} \mathrm{BM}$ 的膜表面的规整 程度与之相比，有着明显的缺陷，因此，我们将在该基 础上进一步对 Pc-PDI-Pc/ $\mathrm{PC}_{61} \mathrm{BM}$ 进行优化.

\section{3 小结}

合成了两端含有氨基官能团的 $N, N^{\prime}$-二(3-氨基丙 基)-1,6,7,12-四(4-叔丁基苯氧基)-3,4:9,10-芢二酰亚胺 (6), 同时选取前期工作中所合成的含羧基酞菁:2(3),

表 1 太阳能电池的各项实验数据

Table 1 Photovoltaic performance of OSC devices

\begin{tabular}{cccccr}
\hline Pc-PDI-Pc $:$ PCBM & $V_{\text {oc }} / \mathrm{V}$ & $J_{\mathrm{sc}}\left(\mathrm{mA} \cdot \mathrm{cm}^{-2}\right)$ & $\mathrm{FF} / \%$ & $\mathrm{PCE} / \%$ & $\mathrm{Rev}^{-}\left(\mathrm{r} \bullet \mathrm{min}^{-1}\right)$ \\
\hline $1: 2.0$ & 0.462 & 0.053 & 17.4 & 0.0043 & 2000 \\
$1: 2.0$ & 0.488 & 0.063 & 17.8 & 0.0055 & 1500 \\
$1: 2.0$ & 0.498 & 0.074 & 20.1 & 0.0074 & 800 \\
$1: 1.0$ & 0.415 & 0.042 & 18.0 & 0.0031 & 2000 \\
$1: 1.0$ & 0.403 & 0.054 & 19.0 & 0.0041 & 1500 \\
$1: 1.0$ & 0.488 & 0.083 & 18.5 & 0.0074 & 800 \\
$1: 0.5$ & 0.421 & 0.056 & 19.8 & 0.0046 & 2000 \\
$1: 0.5$ & 0.575 & 0.057 & 21.7 & 0.0071 & 1500 \\
$1: 0.5$ & 0.472 & 0.104 & 18.2 & 0.0090 & 800 \\
\hline
\end{tabular}




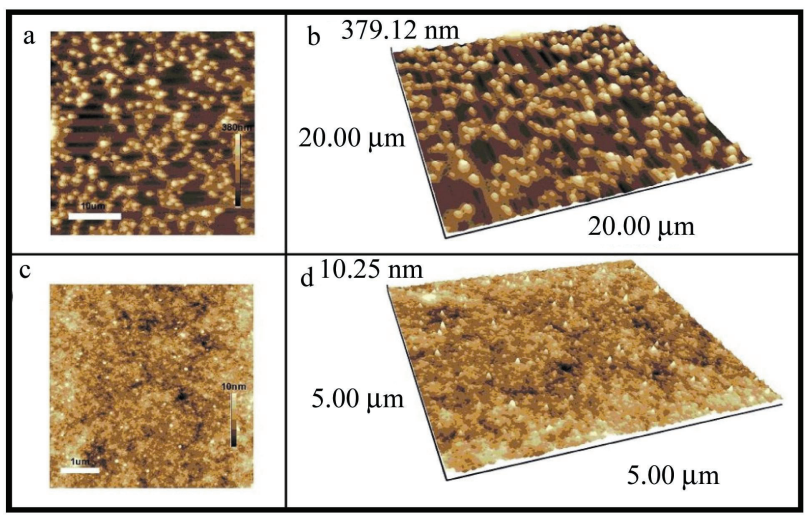

图 5 有机活性层的原子力显微镜

Figure 5 Atomic force microscope of the organic photoactive layer

Pc-Pdi-Pc/PCBM (a and b), P3HT/PCBM (c and d)

9(10),16(17)-三-(对叔丁基苯氧基)-23(24)-(丁二酸单酸 酰胺基)-酞菁锌(8), 通过酰胺键缩合合成了酞菁-菲-酞 菁分子异质结, 并用紫外光谱和荧光光谱研究其光物理 性质，紫外吸收光谱表明该分子的吸收峰在 $300 \sim 780$ $\mathrm{nm}$ 之间, 是唒菁和菲二酰亚胺的吸收峰的叠加结果. 所 得到的酞菁-芢-酞菁分子异质结(9)可以溶液加工, 制 备得到的体异质结型有机太阳能电池的转换效率仅为 $0.009 \%$, 这是由于该分子的成膜性不佳, 后续的分子结 构优化正在进行中.

\section{References}

[1] Zhan, C. L.; Li, A. D. Q. Curr. Org. Chem. 2011, 15, 131.

[2] Chen, Z.; Lohr, A.; Saha-Möller, C. R.; Würthner, F. Chem. Soc. Rev. 2009, 38, 564.

[3] Zang, L.; Che, Y.; Moore, J. S. Acc. Chem. Res. 2008, 41, 1596.

[4] Ke, D. M.; Zhan, C. L.; Xu, S. P.; Ding, X. L.; Peng, A. D.; Sun, J.; He, S. G.; Li, A. D. Q.; Yao, J. N. J. Am. Chem. Soc. 2011, 133, 11022.

[5] Schmidt, R.; Oh, J. H.; Sun, Y.-S.; Deppisch, M.; Krause, A.-M.; Radacki, K.; Braunschweig, H.; Könemann, M.; Bao, P.; Erk, Z. Würthner, F. J. Am. Chem. Soc. 2009, 131, 6215.

[6] Ke, D. M.; Zhan, C. L.; Li, A. D. Q.; Yao, J. N. Angew. Chem., Int. Ed. 2011, 50, 3715.

[7] Schmidt-Mende, L.; Fechtenkötter, A.; Würthner K.; Moons, E.; Friend, R. H.; MacKenzie, J. D. Science 2001, 293, 1119.

[8] Shi, M.-M.; Chen, H.-Z.; Sun, J.-Z.; Ye, J.; Mang, W. Chem. Commun. 2003, 1710.

[9] Li, J.-L.; Dierschke, F.; Wu, J.-S.; Grimsdale, A. C.; Würthner, K. J. Mater. Chem. 2006, 16, 96.

[10] Shin, W. S.; Jeong, H.-H.; Kim, M.-K.; Jin, S.-H.; Kim, M.-R.; Lee, J.-K.; Lee, J. W.; Gal, Y.-S. J. Mater. Chem. 2006, 16, 384.

[11] Mo, X.; Chen, H.-Z.; Wang, Y.; Shi, M.-M.; Wang, M. J. Phys. Chem. B 2005, 109, 7659.
[12] Weitz, R.; Amsharov, T. K.; Zschieschang, U.; Villas, E. B.; Goswami, D. K.; Burghard, M.; Dosch, H.; Jansen, M.; Kern, K.; Klauk, H. J. Am. Chem. Soc. 2008, 130, 4637.

[13] Jones, B. A.; Facchetti, A.; Wasielewski, M. R.; Marks, T. J. J. Am. Chem. Soc. 2007, 129, 15259.

[14] Li, Y.; Tan, L.; Wang, Z.-H.; Qian, H.-L.; Shi, Y.-B.; Hu, W.-P. Org. Lett. 2008, 10, 529.

[15] Hill, Z. B.; Rodovsky, D. B.; Legera, J. M.; Bartholomew, G. P. Chem. Commun. 2008, 6594.

[16] Chen, H. Z.; Ling, M. M.; Mo, X.; Shi, M. M.; Wang, M.; Bao, Z. Chem. Mater. 2007, 19, 816.

[17] Mack, J.; Kobayashi, N. Chem. Rev. 2011, 2, 281.

[18] Claessens, C. G.; Blau, W. J.; Cook, M.; Hanack, M.; Nolte, R. J. M.; Torres, T.; Wöhrle, D. Monatsh. Chem. 2001, 132, 3.

[19] Kolarova, H.; Tomankova, K.; Bajgar, R.; Kolar, P.; Kubinek, R. Ultrasound Med. Biol. 2009, 35, 1397.

[20] Nunes, S. M. T.; Sguilla, F. S.; Tedesco, A. C. Braz. J. Med. Biol. Res. 2004, 37, 273.

[21] Troshin, P. A.; Koeppe, R.; Peregudov, A. S.; Peregudova, S. M.; Egginger, M.; Lyubovskaya, R. N.; Sariciftci, N. S. Chem. Mater. 2007, 19, 5363.

[22] Fischer, M. K. R.; López-Duarte, I.; Wienk, M. M.; Martínez-Díaz, M. V.; Janssen, R. A. J.; Bäuerle, P.; Torres, T. J. Am. Chem. Soc. 2009, 131, 8669.

[23] de la Escosura, A.; Martínez-Díaz, M. V.; Barberá, J.; Torres, T. J. Org. Chem. 2008, 73, 1475.

[24] Kojima, T.; Honda, T.; Ohkubo, K.; Shiro, M.; Kusukawa, T.; Fukuda, T.; Kobayashi, N.; Fukuzumi, S. Angew. Chem., Int. Ed. 2008, 47, 6712.

[25] Zhao, C.; Li, R.; Li, X.; Jiang, J. J. Org. Chem. 2007, 72, 2402.

[26] Yu, X.-W.; Zhan, C.-L.; Huang, Y. Chin. J. Org. Chem. 2012, 32, 770 (in Chinese). (俞孝伟, 詹传郎, 黄彦, 有机化学, 2012, 32, 770.)

[27] Chao, C. C.; Leung, M. K.; Su, O. Y.; Chiu, K. Y.; Shieh, S. J.; Lin, S. C. J. Org. Chem. 2005. 70, 4323.

[28] Chen, Z.; Baumeister, U.; Tschierske, C.; Würthner, F. Chem. Eur. J. 2007, 13, 450 .

[29] Chen, Z.; Stepanenko, V.; Dehm, V.; Prins, P.; Li, D. A.; Seibt, J.; Marquetand, P.; Engel, V.; Würthner, F. Chem. Eur. J. 2007, 13, 436.

[30] Würthner, F.; Thalacker, C.; Diele, S.; Tschierske, C. Chem. Eur. J. 2001, 7, 2245.

[31] Fan, L.; Xu, Y.; Tian, H. Tetrahedron Lett. 2005, 46, 4443.

[32] Li, Y. J.; Wang, N.; Gan, H. Y.; Liu, H. B.; Li, H.; Li, Y. L.; He, X. R.; Huang, C. S.; Gui, S.; Wang, S.; Zhu, D. B. J. Org. Chem. 2005 , $70,9686$.

[33] Xu, S. P.; Sun, J.; Ke, D. M.; Song, G.; Zhang, W.; Zhan, C. L. J. Colloid Interface Sci.2010, 349, 142.

[34] Céspedes-Guirao, F. J.; Ohkubo, K.; Fukuzumi, S.; Sastre-Santos, Á.; Fernández-Lázaro, F. J. Org. Chem. 2009. 74, 5871.

[35] Li, Y. F.; Cao, Y.; Gao, J.; Wang, D. L.; Yu, G. Heeger, A. J. Synth. Met. 1999, 99, 243.

[36] Scharber, M. C.; Mühlbacher, D.; Koppe, M.; Denk, P.; Waldauf, C.; Heeger, A. J.; Brabec, C. J. Adv. Mater. 2006, 18, 789.

[37] Padinger, F.; Rittberger, R. S.; Sariciftc, I. N. S. Adv. Funct. Mater. 2003, 13, 85 . 